\title{
Stichwörter im FamR-Verfahren in alphabetischer Reihenfolge (3. Teil)
}

\begin{tabular}{|c|c|c|c|}
\hline & Seite & & Seit \\
\hline A & & Aufhebung des Annahmeverhältnisses & \\
\hline Abänderung & 1233 & $\rightarrow$ Adoptionssachen & \\
\hline Abfindung & & Aufrechnung $\ldots \ldots \ldots$ & 1287 \\
\hline$\rightarrow$ Unterhaltsverzicht & & Auseinandersetzung der Gütergemein- & \\
\hline Abstammungssachen & 1237 & schaft & \\
\hline Abtrennung aus dem Verbund & & $\rightarrow$ Güterrechtssachen & \\
\hline$\rightarrow$ Verbund & & Ausgleich von Kapitalzahlungen, An- & \\
\hline $\begin{array}{l}\text { Abtretung von Versorgungsansprüchen } \\
\rightarrow \text { Versorgungsausgleichssachen }\end{array}$ & & $\begin{array}{l}\text { spruch auf Abfindung } \\
\rightarrow \text { Versorgungsausgleichssachen }\end{array}$ & \\
\hline Adoptionssachen . . . . . . . . . & 1244 & Ausgleichsansprüche nach der Scheidung & \\
\hline Anerkenntnis & & $\rightarrow$ Versorgungsausgleichssachen & \\
\hline $\begin{array}{l}\rightarrow \text { Anerkenntnis und Anerkenntnis- } \\
\text { urteil (ZPO-Teil) }\end{array}$ & & $\begin{array}{l}\text { Auskunft . . . . . . . . . . . . . . . . } \\
\text { Auskunft über die persönlichen }\end{array}$ & 1288 \\
\hline Anerkennung anderer ausländischer & & Verhältnisse des Kindes . . . . . . . . . & 120 \\
\hline
\end{tabular}

Entscheidungen als Ehesachen . . . . . 1249

Anerkennung ausländischer Entscheidun-

gen in Ehesachen . . . . . . . . . . 1253

Anerkennung ausländischer Entscheidun-

gen nach dem AdWirkG . . . . . . . 1259

Annahme als Kind

$\rightarrow$ Adoptionssachen

Anordnungen nach PsychKG

$\rightarrow$ Unterbringungssachen Minderjähriger (freiG-Teil)

$\rightarrow$ Unterbringungssachen Volljähriger (freiG-Teil)

$\rightarrow$ Kindschaftssachen

Anpassung wegen Invalidität, Tod, Unter-

halt . . . . . . . . . . . . 1262

Antrag und Widerantrag . . . . . . . . . 1263

Antragshäufung

$\rightarrow$ Mehrere Ansprüche (Antragshäufung)

$\rightarrow$ Mehrere Ansprüche (Klagehäufung) (ZPO-Teil)

Anwendung ausländischen Rechts in Ehesachen

$\rightarrow$ Auslandsbezug

$\rightarrow$ Ehesachen

Arrest . . . . . . . . . . . . . . . . . . . . 1264

Aufenthaltsbestimmungsrecht

$\rightarrow$ Bestimmte Kindschaftssachen

$\rightarrow$ Elterliche Sorge

$\rightarrow$ Kindschaftssachen

Auffangwert . . . . . . . . . . . . . . . 1271

Aufgaben nach dem Jugendgerichtsgesetz. 1281

Aufhebung der Ehe . . . . . . . . . . . . . 1285

Aufhebung der Gütergemeinschaft

$\rightarrow$ Güterrechtssachen

\section{B}

Beerdigungskosten

$\rightarrow$ Unterhaltssachen

Befreiung vom Eheverbot

$\rightarrow$ Adoptionssachen

Befreiung von einer gesetzlichen Unter-

haltspflicht . . . . . . . . . . . . 1302

Befreiung von einer Verbindlichkeit . . . . 1303

Begrenztes Realsplitting . . . . . . . . . 1305

Beschleunigungsrüge und -beschwerde . . 1308

Beschränkung oder Wegfall des Versorgungsausgleichs

$\rightarrow$ Versorgungsausgleichssachen

Beschwerde . . . . . . . . . . . . . 1310

Bestimmte Kindschaftssachen . . . . . . . 1313

Beweisverfahren

$\rightarrow$ Selbstständiges Beweisverfahren

Bewilligung oder Aufhebung von Verfahrenskostenhilfe

$\rightarrow$ Verfahrenskostenhilfe

$\rightarrow$ Prozesskostenhilfe (ZPO-Teil)

Bürgschaft

$\rightarrow$ Befreiung von einer Verbindlichkeit

D

Darlehensaufnahme, Genehmigung

$\rightarrow$ Genehmigung einer Erklärung oder deren Ersetzung

Dynamisierter Unterhalt

$\rightarrow$ Unterhaltssachen 
$\rightarrow$ Vereinfachtes Verfahren auf Festsetzung des Unterhalts Minderjähriger

\section{E}

Ehegattenmitarbeit aufgrund Arbeitsver-

trag . . . . . . . . . . . . 1315

Ehesachen . . . . . . . . . . . . . 1316

Ehestörungsklage

$\rightarrow$ Sonstige Familiensachen

Ehevertrag . . . . . . . . . . . . . 1357

Ehewohnungssachen . . . . . . . . . . . . . 1359

Eidesstattliche Versicherung . . . . . . . . . 1370

Einbenennungsverfahren

$\rightarrow$ Bestimmte Kindschaftssachen

$\rightarrow$ Elterliche Sorge

$\rightarrow$ Kindschaftssachen

Einsicht in Abstammungsgutachten

$\rightarrow$ Abstammungssachen

Einstweilige Anordnung . . . . . . . . . . 1372

Elterliche Sorge . . . . . . . . . . . . 1386

Ersetzung der Einwilligung in eine genetische Abstammungsuntersuchung

$\rightarrow$ Abstammungssachen

Ersetzung der Einwilligung zur Annahme als Kind

$\rightarrow$ Adoptionssachen

Ersetzung der Genehmigung einer Erklärung

$\rightarrow$ Genehmigung einer Erklärung oder deren Ersetzung

Ersetzung der Zustimmung des anderen

Ehegatten durch das Familiengericht

bei der Verhandlung des Gesamtguts

$\rightarrow$ Genehmigung einer Erklärung oder deren Ersetzung

Ersetzung der Zustimmung durch das Familiengericht bei einer Verfügung über das Vermögen im Ganzen

$\rightarrow$ Genehmigung einer Erklärung oder deren Ersetzung

Erwerb oder Veräußerung eines Erwerbsgeschäfts des Minderjährigen

$\rightarrow$ Genehmigung einer Erklärung oder deren Ersetzung

\section{F}

Fälligkeit . . . . . . . . . . . . . 1403

Familienrechtlicher Ausgleichsanspruch . . 1404
Feststellung des Bestehens oder Nicht-

bestehens einer Ehe . . . . . . . . . . . 1406

Feststellung des Bestehens oder Nicht-

bestehens der elterlichen Sorge

$\rightarrow$ Elterliche Sorge

Feststellungsverfahren . . . . . . . . . . . . 1407

Früchte

$\rightarrow$ Früchte (ZPO-Teil)

\section{$\mathrm{G}$}

Geldforderung . . . . . . . . . . . 1410

Genehmigung . . . . . . . . . . . . . 1418

Genehmigung einer Erklärung oder deren

Ersetzung . . . . . . . . . . . . . . 1419

Gesamtschuldnerausgleichsanspruch . . . . 1425

Geschäfte über das Vermögen im Ganzen

$\rightarrow$ Genehmigung einer Erklärung oder deren Ersetzung

Geschenke

$\rightarrow$ Sonstige Familiensachen

Gewaltschutzsachen . . . . . . . . . . . 1426

Gleichgeschlechtliche Lebenspartner-

schaftssachen . . . . . . . . . . . . 1435

Grundbuchberichtigungsanspruch

$\rightarrow$ Grundbuchberichtigung (ZPO-Teil)

Grundstücksgeschäfte des Kindes

$\rightarrow$ Genehmigung einer Erklärung oder deren Ersetzung

Gütergemeinschaft (Auseinandersetzung)

$\rightarrow$ Güterrechtssachen

Güterrechtssachen . . . . . . . . . . . . . . . 1437

H

Haftungsfreistellung

$\rightarrow$ Befreiung von einer gesetzlichen

Unterhaltspflicht

$\rightarrow$ Befreiung von einer Verbindlichkeit

$\rightarrow$ Befreiung von einer Verbindlichkeit (ZPO-Teil)

Haupt- und Hilfsantrag

$\rightarrow$ Hilfsantrag

$\rightarrow$ Hilfsantrag (ZPO-Teil)

Haushaltssachen . . . . . . . . . . . . . . 1442

Haustiere . . . . . . . . . . . . . . . . . 1450

Hilfsantrag . . . . . . . . . . . . . . . . 1452

Hilfsaufrechnung

$\rightarrow$ Aufrechnung

$\rightarrow$ Aufrechnung (ZPO-Teil) 
J

Jugendamtsurkunde

$\rightarrow$ Abänderung

$\rightarrow$ Unterhaltssachen

Jugendgerichtsgesetz

$\rightarrow$ Aufgaben nach dem Jugendgerichtsgesetz

\section{K}

Kapitalabfindung

$\rightarrow$ Unterhaltsverzicht

Kindergeldabzug

$\rightarrow$ Kindergeldbezugsberechtigung

$\rightarrow$ Unterhaltssachen

$\rightarrow$ Vereinfachtes Verfahren auf Festsetzung des Unterhalts Minderjähriger

Kindergeldbezugsberechtigung . . . . . . . 1453

Kindesherausgabe . . . . . . . . . . 1458

Kindesmutter, Unterhalt

$\rightarrow$ Unterhaltssachen

Kindschaftssachen im Verbund

$\rightarrow$ Verbund

Kindschaftssachen . . . . . . . . . . . . 1468

Klage und Widerklage

$\rightarrow$ Antrag und Widerantrag

Kontenpfändung, europäische (EuKoPfVO) bei Unterhaltsforde-

rungen . . . . . . . . . . . . . . . . . 1469

Kosten des Verfahrens

$\rightarrow$ Kosten des Rechtsstreits

Kosten

$\rightarrow$ Nebenforderungen (ZPO-Teil)

Kostenvorschuss für ein gerichtliches Ver-

fahren . . . . . . . . . . . . . . 1472

Künftige Forderungen . . . . . . . . . . . . 1476

1

Lebenspartnerschaftssachen . . . . . . . . 1477

Leistungen nach dem SGB II . . . . . . . . 1477

\section{M}

Mahnverfahren

$\rightarrow$ Mahnverfahren (ZPO-Teil)

Mehrbedarf

$\rightarrow$ Unterhaltssachen

Mehrere Ansprüche (Antragshäufung) . . 1480
Seite

Mehrere Kinder

$\rightarrow$ Kindschaftssachen

Mehrwertvergleich . . . . . . . . . . . . 1482

Mindestwert . . . . . . . . . . . . . 1485

N

Nachforderungsklage

$\rightarrow$ Nachforderungsverfahren

Nachforderungsverfahren . . . . . . . . . 1486

Naturalunterhalt

$\rightarrow$ Unterhaltssachen

Negative Feststellungsklage

$\rightarrow$ Feststellungsverfahren

$\rightarrow$ Feststellungsklage (ZPO-Teil)

Nutzungen

$\rightarrow$ Nutzungen (ZPO-Teil)

Nutzungsentschädigung bei Ehewoh-

nungssachen

$\rightarrow$ Ehewohnungssachen

$\rightarrow$ Nutzungsentschädigung nach

Rechtskraft der Scheidung

Nutzungsentschädigung nach Rechtskraft

der Scheidung . . . . . . . . . . . . . 1487

Nutzungsentschädigung bei Haushalts-

sachen

$\rightarrow$ Haushaltssachen

$\rightarrow$ Nutzungsentschädigung nach Rechtskraft der Scheidung

$P$

Pflegschaften . . . . . . . . . . . . . 1495 Prozesskostenhilfe

$\rightarrow$ Verfahrenskostenhilfe

$\rightarrow$ Prozesskostenhilfe (ZPO-Teil)

Prozesskostenvorschuss

$\rightarrow$ Kostenvorschuss für ein gerichtliches Verfahren

$\mathbf{R}$

Realsplitting

$\rightarrow$ Begrenztes Realsplitting

Rechtsbeschwerde . . . . . . . . . . . . . 1496

Rechtsmittelverfahren . . . . . . . . . . 1498

Religiöse Kindererziehung

$\rightarrow$ Elterliche Sorge

Rückzahlung geleisteten Unterhalts . . . 1505 
Inhaltsverzeichnis | FamR-Stichwörter

Rückabwicklung von Zuwendungen

$\rightarrow$ Sonstige Familiensachen

Rückgabeanspruch, Geschenke zwischen

Verlobten

$\rightarrow$ Sonstige Familiensachen

\section{S}

Schadensersatzansprüche des umgangs-

berechtigten Elternteils . . . . . . . . . . 1505

Schadensfreiheitsrabatt . . . . . . . . . 1506

Scheidungssache . . . . . . . . . . . . 1509

Schuldrechtlicher Versorgungsausgleich . . 1510

Selbständiges Beweisverfahren . . . . . . . 1510

Sicherheitsleistung . . . . . . . . . 1513

Sonstige Familiensachen . . . . . . . . . . . 1514

Sorgerecht

$\rightarrow$ Bestimmte Kindschaftssachen

$\rightarrow$ Elterliche Sorge

Sozialrechtliche Verfahren, verwaltungsrechtliche Verfahren bei Versorgungs-

ausgleich . . . . . . . . . . . . . . . . 1520

Sprungrechtsbeschwerde, Zulassung der . . 1520

Steuererstattungen und Steuerschulden,

Aufteilung von . . . . . . . . . . . . . 1521

Steuerliche Veranlagung

$\rightarrow$ Zusammenveranlagung

Stufenverfahren . . . . . . . . . . . . 1524

Stundung und Zugewinnausgleich

$\rightarrow$ Zugewinngemeinschaft

\section{$\mathrm{T}$}

Teilanerkenntnisbeschluss

$\rightarrow$ Anerkenntnis

$\rightarrow$ Teilurteil (ZPO-Teil)

Titulierung unstreitiger Unterhaltsbeträge

$\rightarrow$ Geldforderung

$\rightarrow$ Unterhaltssachen

Trennungsunterhalt

$$
\rightarrow \text { Unterhaltssachen }
$$

Trennungsverfahren nach italienischem Recht

$\rightarrow$ Ehesachen

\section{U}

Übertragung von Vermögensgegenständen

$\rightarrow$ Zugewinngemeinschaft

Übrige Kindschaftssachen . . . . . . . . . . 1528
Seite

Umgangspflegschaft . . . . . . . . . . . . . 1532

Umgangsrecht . . . . . . . . . . . . . . . . . 1533

Unbenannte Zuwendungen . . . . . . . . . . . 1546

Unbezifferter Leistungsantrag ... . . . . 1547

Unstreitige Sockelbeträge

$\rightarrow$ Geldforderung

Unterhaltsansprüchen, Überleitung von . . 1549

Unterhaltsprivileg . . . . . . . . . . 1550

Unterhaltssachen . . . . . . . . . . . . . . 1550

Unterhaltsverzicht . . . . . . . . . . 1568

V

Vaterschaftsanfechtung/-feststellung

$\rightarrow$ Abstammungssachen

Vaterschaftsfeststellung und Mindestunterhalt

$\rightarrow$ Abstammungssachen

Verbund. . . . . . . . . . . . . . . . . 1569

Vereinfachtes Verfahren auf Festsetzung

des Unterhalts Minderjähriger . . . . . 1583

Verfahrenskostenhilfe . . . . . . . . . . . 1589

Verfassungsbeschwerde . . . . . . . . . . . 1590

Vergleich . . . . . . . . . . . . . . . 1592

Verlöbnis

$\rightarrow$ Sonstige Familiensachen

Vermittlungsverfahren ... . . . . . . . . 1595

Versorgungsausgleichssachen . . . . . . . . 1599

Vertraglicher Unterhalt . . . . . . . . . . . . 1621

Verzicht, Unterhalt

$\rightarrow$ Unterhaltsverzicht

Vollstreckbarerklärung ausländischer

Entscheidungen . . . . . . . . . . . 1623

Vollstreckung . . . . . . . . . . . . . . . 1630

Vollstreckungsabwehrantrag . . . . . . 1637

Vorläufiger Rechtsschutz in Familien-

sachen . . . . . . . . . . . . . . . . . . 1640

Vormundschaften ... . . . . . . . . . 1641

W

Wiederherstellung des ehelichen Lebens

$\rightarrow$ Ehesachen

$\rightarrow$ Sonstige Familiensachen

Vorzeitiger Zugewinnausgleich

$\rightarrow$ Zugewinngemeinschaft

Wechselseitige Abänderungsanträge . . . 1645

Wiederkehrende Leistungen . . . . . . . . . . 1646

Wohnungszuweisungssachen

$\rightarrow$ Ehewohnungssachen 


\section{FamR-Stichwörter | Inhaltsverzeichnis}

Z

Zahlung

$\rightarrow$ Geldforderung

Zahlungsvereinbarung

$\rightarrow$ Zahlungsvereinbarung (ZPO-Teil)

Zeitpunkt der Wertberechnung . . . . . . 1647

Zinsen . . . . . . . . . . . . . . . . . . . . . 1652
Seite

Zugewinngemeinschaft . . . . . . 1653

Zusammenveranlagung . . . . . . . . 1663

Zustimmung .............. 1665

Zwangsvollstreckung

$\rightarrow$ Vollstreckung

$\rightarrow$ Zwangsvollstreckung

(ZPO-Teil) 